\title{
Numerical pricing of Coco bonds with Parisian trigger feature using the Fortet method
}

\author{
Chi Man Leung \\ Department of Mathematics, \\ Hong Kong Baptist University, Hong Kong, China \\ Yue Kuen Kwok ${ }^{1}$ \\ Department of Mathematics, \\ Hong Kong University of Science and Technology, Hong Kong, China
}

\begin{abstract}
Unlike conventional convertible bonds, contingent convertible ( $\mathrm{CoCo}$ ) bonds are converted into equity shares of the issuing bank subject to certain trigger mechanisms (accounting and/or regulatory trigger) when the issuing bank is under financial non-viable state. We consider pricing of these CoCos using the contingent claims approach, where the state variables are the stock price and Tier 1 capital ratio. We use the Parisian feature to model the regulatory trigger where equity conversion is triggered when the capital ratio stays under the non-viable state cumulatively for a certain period of time. The accounting trigger is modeled using the one-touch barrier feature associated with the capital ratio. The Parisian trigger feature adds one extra path dependent state variable in the pricing model of a CoCo bond. We design effective numerical algorithms for pricing the Coco bonds using the extended Fortet method that avoid the issuance of adding one state variable for the Parisian feature of regulatory trigger. Pricing properties of the CoCo bonds under both regulatory trigger and accounting trigger are explored.
\end{abstract}

Keywords: Coco bonds, conversion triggers, Parisian feature, Fortet method

\section{Introduction}

The contingent convertible ( $\mathrm{CoCo}$ ) bonds are hybrid equity-credit securities that are designed to have the loss absorption mechanism, where the CoCo bond is converted into risky equity when the issuing bank is under financial non-viable state. This equity conversion feature serves the primary purpose of the CoCo bonds as a readily available source of bank capital when the bank is in the state of non-viability. Another loss absorption mechanism, though less common, is via predefined principal writedown of the principal. Since their first launch in 2009, the growth of the issuance of CoCo bonds has been quite phenomenal, with issuance amount more than 100 billion by 2014. From the investors' perspective, CoCo bonds pay high coupon rate that compensates the potential loss absorption liabilities. From the issuer's

\footnotetext{
${ }^{1}$ Correspondence author; email: maykwok@ust.hk
} 
perspective, the CoCo bonds can be counted as regulatory capital even under the more stringent regulatory requirement imposed after the financial tsunami in 2008. Regulators have been demanding more loss absorbency within hybrid capital, in the view that the holders of these capital instruments can contribute to the recovery phase of a distressed firm.

The loss absorption mechanism is activated either by regulator's discretion based on judgment about solvency prospects of the issuing bank and / or mechanical trigger based on the capital ratio that falls below some preset threshold value. The capital ratio trigger can be either set contractually in terms of the book value of the Common Equity Tier 1 capital as a ratio of the risk-weighted assets or the market value of the ratio of the bank's stock market capitalization to its assets. The details of these trigger mechanisms can be found in Avdjiev et al. (2013). There are subtleties in the actual implementation of these trigger mechanisms. Due to the general lack of transparency on the criteria used by regulators in activating the discretionary trigger, the timing of the activation is highly uncertain. There may be a lagged effect in the activation of the book value trigger since accounting reports are at most published quarterly. The market trigger may be activated in a more timely fashion since it is based on observed market information. However, the use of market trigger may lead to the so-called death spiral effect of falling share price when the issuing bank is in distress state. This arises from the dynamic hedging of equity exposure of CoCo bond investors via offsetting short position in the underlying shares. Glasserman and Nouri (2012) present a thorough discussion on the risk implication of CoCo bonds under alternative conversion mechanisms.

The pricing of CoCo bonds poses great challenge, in particular, the modeling of uncertainty of the timing of conversion trigger. Wilkens and Bethke (2014) present an empirical assessment of selected pricing models of CoCo bonds. They categorize the pricing models into three types: structural models, equity derivatives models and credit derivatives models. The structural models use the fundamental information of issuing banks asset and liabilities structure, and impose contingent capital conversion into equity when certain trigger mechanism is activated. However, equity value is an option on the firm value, so optionality on equity value becomes a compound option on the firm value process. Brigo et al. (2015) use the structural-default approach that considers two different thresholds for the firm value, the first one triggering equity conversion and the second one triggering default. On the other hand, Wilkens and Bethke (2014) advocate the use of equity derivatives models due to its straightforward modeling and interpretation of the conversion mechanism. For example, Spiegeleer and Schoutens (2012) model conversion trigger by the first time that the stock price crosses a barrier level from above in their equity derivatives model. In general, equity derivatives models have better tractability due to its simplicity as one-dimensional pricing models since the stock price is the only single state variable. However, this may not reflect the contractual reality in CoCo bonds where conversion into equity is triggered based on the capital ratio falling below a preset threshold. In the credit derivatives models, conversion trigger is based on the credit spreads or the risk premia of credit default swaps (CDS). Pelger (2012) argue that defining the conversion trigger in terms of credit spreads or CDS premia may circumvent the unobservability of the firm value process. With the advantage of rapid adjustment to new information, credit spreads and CDS premia reflect new changes 
in the firm value in a timely fashion. Using the reduced form approach, Cheridito and Xu (2014) model the arrival of the conversion trigger in a CoCo bond by the first jump of a time-changed Poisson process.

In this paper, we propose a two-state pricing model using the joint stochastic process of the Tier 1 capital ratio and stock price as the underlying state variables. The capital ratio is included in the pricing model since it appears as part of the contractual specification. Under Basel III, the minimum trigger level in terms of the ratio of Common Equity Tier 1 capital to risk-weighted assets required for a $\mathrm{CoCo}$ bond to be qualified as Additional Tier 1 capital is $5.125 \%$. Many CoCo bonds have set their trigger levels at this value. Since equity conversion occurs upon activation of trigger, so it is more direct to use the stock price as the underlying state variable rather than the firm value process. We would like to incorporate both the mechanical trigger and regulatory trigger in our Coco bond pricing model. Mechanical trigger is activated when the capital ratio hits the one-touch threshold level from above. The regulatory trigger is modeled by the event of breaching the allowable cumulative occupation time that the capital ratio is staying below some threshold value that warns against potential non-viability of the issuing bank. In our CoCo bond pricing model, we set the warning threshold to be above the mechanical trigger threshold of mandatory equity conversion. The regulatory trigger mechanism resembles the cumulative Parisian feature on the knock-out condition in barrier style derivatives. The dimensionality of the pricing model increases by one due to an inclusion of this extra Parisian state variable.

In the design of an effective numerical algorithm for computing the fair value of a CoCo bond, we adopt the Fortet method that was first used for pricing defaultable debts under stochastic interest rates (Longstaff and Schwartz, 1995; Collin-Dufresne and Goldstein, 2001). Later, the Fortet method was extended by Bernard et al. (2005, 2006, 2008) in their series of papers on pricing various types of barrier style derivatives under stochastic interest rates. Another nice application of the Fortet method is presented by Coculescu et al. (2008) in pricing defaultable sensitive claims under imperfect information. The Fortet method solves an integral equation for the determination of the first passage time to a barrier under the joint Gaussian process by making use of the strong Markov property of the underlying Gaussian processes. We manage to extend the Fortet method in pricing a CoCo bond in two aspects. Firstly, we deal with two barriers, the upper warning threshold and the lower mandatory trigger threshold on the capital ratio. Secondly, the curse of an additional Parisian state variable in the CoCo bond pricing model is resolved. The computational complexity of our extended Fortet method remains to be the same as that of the pricing algorithm for pricing one-touch single-barrier options under joint Gaussian process.

The paper is organized as follows. In the next section, we use the equity derivatives approach to construct the pricing model of a CoCo bond under both mechanical and regulatory trigger mechanisms. The stochastic process of the Tier 1 capital ratio is modeled as a mean reversion Geometric Brownian motion that is correlated with the Geometric Brownian motion of the stock price process. The pricing model includes the usual cash flows of coupons and terminal par payment, together with the feature of conversion into equity under the two conversion mechanisms. The equity conversion is activated either under the one-touch barrier criterion (mechanical trigger) or the Parisian cumulative occupation time criterion (regulatory trigger) based on the path realization of the stochastic process of the Tier 1 cap- 
ital ratio. In Section 3, we consider an extension of the Fortet method to devise an effective numerical algorithm for pricing a CoCo bond with the Parisian feature and one-touch barrier feature of equity conversion. We achieve dimension reduction in the pricing algorithm via the determination of the auxiliary CoCo bond value function at the Tier 1 capital ratio that is slightly below the warning threshold of non-viability. In Section 4, we present numerical pricing results and performance analysis of the algorithm. We also present studies of the pricing properties of the CoCo bonds under various market conditions. Summary and conclusive remarks are presented in the last section.

\section{Model formulation}

Similar to the pricing model of conventional convertible bonds, the value of a CoCo bond is decomposed into the bond component and equity component. Unlike conventional convertible bonds where conversion into equity occurs when the underlying stock price is performing well, conversion into shares of the issuing bank in a CoCo bond occurs when the Tier 1 capital ratio either stays below the warning threshold $G$ consecutively for a period of $d$ (years) or hits an one-touch conversion barrier level $B$, where $B<G$. These two trigger mechanisms of mandatory equity conversion are called the Parisian trigger and one-touch trigger, respectively. Provided that no trigger event is activated throughout the contractual life of the CoCo bond, the CoCo investor is entitled to receive the bond par $F$ at bond's maturity and the coupon payment stream at the annualized rate of $c$ for the whole life of the bond. When the CoCo bond is terminated prematurely due to either one of the two conversion triggers, the bond is converted into $N$ shares of the underlying stock. It is common to set a floored value $K$ on the stock price at equity conversion so that the Coco bond value upon conversion has a lower floor value.

Let $S_{t}$ and $Y_{t}$ denote the price process of the underlying stock and the logarithm of the value process of the Tier 1 capital ratio, respectively. Under a risk neutral measure $Q$, the dynamics of $S_{t}$ and $Y_{t}$ are assumed to evolve as follows:

$$
\begin{aligned}
& \frac{d S_{t}}{S_{t}}=(r-q) d t+\sigma\left(\sqrt{1-\rho^{2}} d W_{t}^{1}+\rho d W_{t}^{2}\right), \\
& d Y_{t}=\alpha\left(\mu-Y_{t}\right) d t+\sigma_{Y} d W_{t}^{2}
\end{aligned}
$$

where $S_{t}$ follows the usual Geometric Brownian motion and $Y_{t}$ follows the mean reversion process with mean reversion level $\mu$ and reversion rate $\alpha, W_{t}^{1}$ and $W_{t}^{2}$ are two independent standard Brownian motions. Here, the correlation coefficient $\rho$, riskfree interest rate $r$, dividend yield $q$, stock price volatility $\sigma$, Tier 1 capital volatility $\sigma_{Y}, \mu$ and $\alpha$ are assumed to be constant.

We let $T$ be the maturity date of the Coco bond and time zero be the time of initiation of the Coco bond. At issuance of the Coco bond, the issuing bank should not be under the non-viable state; so we observe $Y_{0}>\ln G>\ln B$. One-touch trigger occurs at the first random time $\tau_{B}$ at which $Y_{t}$ hits the logarithm of the lower barrier $\ln B$ from above; that is,

$$
\tau_{B}=\inf \left\{s \geq 0: Y_{s}=\ln B \mid S_{0}, Y_{0}\right\} .
$$


On the other hand, the monitoring of $Y_{t}$ staying below the warning threshold $\ln G$ is initiated at the random time that $Y_{t}$ hits $\ln G$ from above; and monitoring is discontinued once $Y_{t}$ moves above $\ln G$. Regulatory equity conversion (Parisian trigger) occurs when $Y_{t}$ stays below $\ln G$ consecutively for a period of $d$ years, conditional on $Y_{t}$ staying above $\ln B$ throughout. Let $H_{t, \ln G}$ denote the excursion time that the $\log$ capital ratio process $Y_{t}$ stays below $\ln G$ in its most recent excursion to the region below $\ln G$; that is,

$$
H_{t, \ln G}=\left(t-h_{t, \ln G}\right) \mathbf{1}_{\left\{Y_{t} \leq \ln G\right\}},
$$

where

$$
h_{t, \ln G}=\sup \left\{u \leq t: Y_{u}=\ln G\right\} .
$$

Given the time period $d$, the right-continuous inverse of the excursion time is defined by

$$
\tau_{P}=\inf \left\{t \geq 0: H_{t, \ln G} \geq d\right\}
$$

That is, $\tau_{P}$ is the first random time at which the excursion time to the region below $\ln G$ reaches $d$ years so that the Parisian trigger occurs. As a summary, the Coco bond terminates due to one-touch trigger when $\tau_{B}<\min \left\{\tau_{P}, T\right\}$ and regulatory equity conversion occurs provided that $\tau_{P}<\min \left\{\tau_{B}, T\right\}$. The Coco bond survives until maturity $T$ provided that $T<\min \left\{\tau_{P}, \tau_{B}\right\}$.

We let $c_{i}$ be the discrete coupon amount received on the coupon payment date $t_{i}, i=$ $1,2, \cdots, n$, where $c_{i}=c F\left(t_{i}-t_{i-1}\right)$. Here, $c$ denotes the constant annualized coupon rate of the Coco bond. We take $t_{0}=0$ and $t_{n}=T$ for convenience. The discrete coupon $c_{i}$ is received at $t_{i}, i=1,2, \cdots, n$, and the par $F$ is received at $T$, provided that the Coco bond survives at $t_{i}$ and $T$, respectively. The sum of the coupons and par in present value received contingent upon survival of the Coco bond gives the bond component of the Coco bond. The equity component of the bond is given by the present value of the shares received upon mandatory conversion under either the Parisian trigger or one-touch trigger. Under the risk neutral pricing measure $Q$, the time-0 value of the Coco bond is given by

$$
\begin{aligned}
V_{0}\left(S_{0}, Y_{0} ; T\right)=\mathbb{E}_{Q} & {\left[\sum_{i=1}^{n} c_{i} e^{-r t_{i}} \mathbf{1}_{\left\{t_{i}<\min \left\{\tau_{P}, \tau_{B}\right\}\right\}}+e^{-r T} F \mathbf{1}_{\left\{T<\min \left\{\tau_{P}, \tau_{B}\right\}\right\}}\right.} \\
& +N \max \left\{S_{\tau_{P}}, K\right\} e^{-r \tau_{P}} \mathbf{1}_{\left\{\tau_{P}=\min \left\{\tau_{P}, \tau_{B}\right\} \leq T\right\}} \\
& \left.+N \max \left\{S_{\tau_{B}}, K\right\} e^{-r \tau_{B}} \mathbf{1}_{\left\{\tau_{B}=\min \left\{\tau_{P}, \tau_{B}\right\} \leq T\right\}} \mid S_{0}, Y_{0}\right],
\end{aligned}
$$

where $Y_{0}>\ln G>\ln B$ and $K$ is the floored value of the stock price at equity conversion. The determination of the joint density of $\left(\tau_{P}, S_{\tau_{P}}\right)$ poses the major challenge in the pricing calculation of $V_{0}\left(S_{0}, Y_{0} ; T\right)$. We would like to explore an alternative pricing approach to avoid such nuisance. In our subsequent exposition, we prefer to use $X_{t}=\ln S_{t}$ as the state variable, where the dynamics of $X_{t}$ is governed by

$$
d X_{t}=\left(r-q-\frac{\sigma^{2}}{2}\right) d t+\sigma\left(\sqrt{1-\rho^{2}} d W_{t}^{1}+\rho d W_{t}^{2}\right)
$$

We denote the time- $t$ value function of the Coco bond by $V_{t}\left(X_{t}, Y_{t} ; T\right)$. 
Since the Parisian feature based on the excursion time staying below $\ln G$ is activated once the logarithm of capital ratio crosses $\ln G$ from above, it is instructive to define the auxiliary value function of the Coco bond to be $V_{t}^{G}\left(X_{t} ; T\right)$ as follows:

$$
V_{t}^{G}\left(X_{t} ; T\right)=\lim _{\varepsilon \rightarrow 0^{+}} V_{t}\left(X_{t}, \ln G-\varepsilon ; T\right) .
$$

We define the random first passage time of $Y_{s}, s \geq t$, that reaches an infinitesimally small distance $\varepsilon$ below the warning barrier $\ln G$ from above to be

$$
\tau_{G}^{-}=\inf \left\{s \geq t: Y_{s}=\ln G-\varepsilon \mid X_{t}, Y_{t}\right\}, \quad Y_{t}>\ln G .
$$

The key ingredient in our pricing formulation is to express $V_{t}\left(X_{t}, Y_{t} ; T\right)$ in terms of $V_{t}^{G}\left(X_{t} ; T\right)$ and payments of coupons and par contingent on survival. The pricing calculations mainly involve the joint density of $\left(\tau_{G}^{-}, X_{\tau_{G}^{-}}\right)$, the numerical approximation of which can be found by the use of the Fortet method. Assuming $Y_{t}>\ln G$, the time- $t$ value of the discrete coupons received within the time period $(t, s]$ is represented by

$$
c(t, s)=\sum_{i=1}^{n} c_{i} e^{-r\left(t_{i}-t\right)} \mathbf{1}_{\left\{t_{i} \in(t, s]\right\}} .
$$

Under the risk neutral measure $Q$, we let $f_{G}^{-}(u, x)$ denote the conditional joint density function of $\left(\tau_{G}^{-}, X_{\tau_{G}^{-}}\right)$, where

$$
f_{G}^{-}(u, x) d u d x=Q\left(\tau_{G}^{-} \in(u, u+d u), X_{\tau_{G}^{-}} \in(x, x+d x) \mid X_{t}, Y_{t}\right)
$$

and the marginal density function of $X_{T}$ conditional on survival up to $T$ by

$$
F_{G}^{-}(x) d x=Q\left(\tau_{G}^{-}>T, X_{T} \in(x, x+d x) \mid X_{t}, Y_{t}\right)
$$

Conditional on $Y_{t}>\ln G$, based on the strong Markov property of the joint process of $X_{t}$ and $Y_{t}$, we deduce that

$$
\begin{aligned}
V_{t}\left(X_{t}, Y_{t} ; T\right)= & \mathbb{E}_{Q}^{t}\left[\left[c(t, T)+e^{-r(T-t)} F\right] \mathbf{1}_{\left\{\tau_{G}^{-}>T\right\}}\right. \\
& \left.+\left[c\left(t, \tau_{G}^{-}\right)+e^{-r\left(\tau_{G}^{-}-t\right)} V_{\tau_{G}^{-}}^{G}\left(X_{\tau_{G}^{-}} ; T\right)\right] \mathbf{1}_{\left\{\tau_{G}^{-} \leq T\right\}}\right] \\
= & \int_{-\infty}^{\infty}\left[c(t, T)+e^{-r(T-t)} F\right] F_{G}^{-}(x) d x \\
& +\int_{t}^{T} \int_{-\infty}^{\infty}\left[c(t, u)+e^{-r(u-t)} V_{u}^{G}(x ; T)\right] f_{G}^{-}(u, x) d x d u, \quad 0 \leq t \leq T,
\end{aligned}
$$

where $\mathbb{E}_{Q}^{t}$ denotes the expectation under $Q$ conditional on $X_{t}$ and $Y_{t}$.

Next, we present the formulation of $V_{t}^{G}\left(X_{t} ; T\right)$. There are 3 possible scenarios to be considered: (i) leaving the monitoring state at a later time before maturity, (ii) mechanical conversion into equity under the one-touch trigger, (iii) regulatory conversion into equity under the Parisian trigger. The quantitative modeling of these 3 scenarios is summarized below. 
1. When the logarithm of capital ratio crosses $\ln G+\varepsilon$ from below prior to the occurrence of the Parisian trigger or one-touch trigger, the Coco bond leaves the monitoring state and the clock of counting the excursion time of staying between $\ln G$ and $\ln B$ is set to be zero. We define

$$
\tau_{G}^{+}=\inf \left\{s \geq t ; Y_{s}=\ln G+\varepsilon \mid X_{t}, Y_{t}=\ln G-\varepsilon\right\} .
$$

Stated mathematically, this scenario occurs when $\tau_{G}^{+}<\min \left\{\tau_{B}, t+d\right\}$ and the value function at $\tau_{G}^{+}$becomes $V_{\tau_{G}^{+}}\left(X_{\tau_{G}^{+}}, \ln G+\varepsilon ; T\right)$.

2. Mechanical conversion into equity occurs at $\tau_{B}$, where $\tau_{B}<\min \left\{\tau_{G}^{+}, t+d\right\}$. The corresponding payoff of the Coco bond at $\tau_{B}$ is $N \max \left\{e^{X_{\tau_{B}}}, K\right\}$.

3. When the Tier 1 capital ratio stays between $G$ and $B$ continuously for a time period of $d$, where $t+d<\min \left(\tau_{B}, \tau_{G}^{+}\right)$, regulatory conversion into equity occurs. The corresponding payoff of the Coco bond at $t+d$ is $N \max \left\{e^{X_{t+d}}, K\right\}$. be

We define the marginal density function of $X_{t+d}$ conditional on $t+d<\min \left\{\tau_{G}^{+}, \tau_{B}\right\}$ to

$$
\left.F_{G^{+}, B}(x) d x=Q\left(t+d<\min \left\{\tau_{G}^{+}, \tau_{B}\right\}\right), X_{t+d} \in(x, x+d x) \mid X_{t}, Y_{t}=\ln G-\varepsilon\right) .
$$

The conditional joint density functions $f_{G^{+}}(u, x)$ and $f_{B}(u, x)$ are similarly defined by

$$
\begin{gathered}
f_{G^{+}}(u, x) d x d u=Q\left(\tau_{G}^{+} \in(u, u+d u), X_{\tau_{G}^{+}} \in(x, x+d x) \mid X_{t}, Y_{t}=\ln G-\varepsilon\right) \mathbf{1}_{\left\{\tau_{G}^{+}<\min \left\{\tau_{B}, t+d\right\}\right\}}, \\
f_{B}(u, x) d x d u=Q\left(\tau_{B} \in(u, u+d u), X_{\tau_{B}} \in(x, x+d x) \mid X_{t}, Y_{t}=\ln G-\varepsilon\right) \mathbf{1}_{\left\{\tau_{B}<\min \left\{\tau_{G}^{+}, t+d\right\}\right\}} .
\end{gathered}
$$

For $t+d<T$, the time- $t$ auxiliary value function $V_{t}^{G}\left(X_{t} ; T\right)$ is then given by

$$
\begin{aligned}
V_{t}^{G}\left(X_{t} ; T\right)= & \int_{t}^{t+d} \int_{-\infty}^{\infty} f_{G}^{+}(u, x)\left[c(t, u)+e^{-r(u-t)} V_{u}(x, \ln G+\varepsilon ; T)\right] d x d u \\
& +\int_{t}^{t+d} \int_{-\infty}^{\infty} f_{B}(u, x)\left[c(t, u)+e^{-r(u-t)} N \max \left\{e^{x}, K\right\}\right] d x d u \\
& +\int_{-\infty}^{\infty} F_{G^{+}, B}(x)\left[c(t, t+d)+e^{-r d} N \max \left\{e^{x}, K\right\}\right] d x
\end{aligned}
$$

Lastly, for $t+d>T$, the regulatory trigger would not occur since the time to maturity is less than the accumulated excursion time required for activating the Parisian trigger. In this case, the pricing formulation is much simplified since only the one-touch trigger remains. We may simply set $\tau_{P}=\infty$ in eq. (2.3) and perform the expectation calculation based on the knowledge of the joint density function of $\left(\tau_{B}, X_{\tau_{B}}\right)$.

There do not exist analytic formulas for the above conditional density functions of the first passage times. In this paper, we employ the extended Fortet method to derive the recursive schemes for calculating the numerical approximation of these conditional density functions (see Section 3). As part of the derivation procedure of the recursive Fortet schemes, 
it is necessary to derive an analytic formula of the distribution function of $Y_{t}$ conditional on varying level of $X_{t}$ that is defined as follows:

$$
L\left(x, y, t ; x_{0}, y_{0}, t_{0}\right) d x=Q\left(Y_{t} \leq y, X_{t} \in(x, x+d x) \mid X_{t_{0}}=x_{0}, Y_{t_{0}}=y_{0}\right) .
$$

Thanks to the joint Gaussian distribution of $X_{t}$ and $Y_{t}$, it can be shown that

$$
L\left(x, y, t ; x_{0}, y_{0}, t_{0}\right)=\frac{e^{-\frac{\left[x-x_{0}-\left(r-q-\frac{\sigma^{2}}{2}\right)\left(t-t_{0}\right)\right]^{2}}{2 \sigma^{2}\left(t-t_{0}\right)}}}{\sqrt{2 \pi \sigma^{2}\left(t-t_{0}\right)}} N\left(\frac{y-\mu_{Y}}{\Sigma_{Y}}\right),
$$

where

$$
\begin{aligned}
\mu_{Y}= & y_{0} e^{-\alpha\left(t-t_{0}\right)}+\mu\left[1-e^{-\alpha\left(t-t_{0}\right)}\right] \\
& +\frac{\frac{\rho \sigma \sigma_{Y}}{\alpha}\left[1-e^{-\alpha\left(t-t_{0}\right)}\right]}{\sigma^{2}\left(t-t_{0}\right)}\left[x-x_{0}-\left(r-q-\frac{\sigma^{2}}{2}\right)\left(t-t_{0}\right)\right], \\
\Sigma_{Y}^{2}= & \sigma^{2}\left(t-t_{0}\right)-\frac{\left\{\frac{\rho \sigma \sigma_{Y}}{\alpha}\left[1-e^{-\alpha\left(t-t_{0}\right)}\right]\right\}^{2}}{\sigma^{2}\left(t-t_{0}\right)} .
\end{aligned}
$$

The derivation of eq. (2.8a) is presented in Appendix A. One can also obtain the analytic expression of an alternative distribution function defined as follows:

$$
\begin{aligned}
M\left(x, y, t ; x_{0}, y_{0}, t_{0}\right) d x & =Q\left(Y_{t}>y, X_{t} \in(x, x+d x) \mid X_{t_{0}}=x_{0}, Y_{t_{0}}=y_{0}\right) \\
& =\frac{e^{-\frac{\left[x-x_{0}-\left(r-q-\frac{\sigma^{2}}{2}\right)\left(t-t_{0}\right)\right]^{2}}{2 \sigma^{2}\left(t-t_{0}\right)}}}{\sqrt{2 \pi \sigma^{2}\left(t-t_{0}\right)}}\left[1-N\left(\frac{y-\mu_{Y}}{\Sigma_{Y}}\right)\right] .
\end{aligned}
$$

The earlier papers by Bernard et al. (2005, 2006, 2007) on pricing barrier options under stochastic interest rates employ the Fortet method to derive the density function of the first passage time to single barrier. Since we have the lower barrier $B$ and upper barrier $G$ in our Coco pricing model, so it is necessary to derive two sets of equations for the individual first passage time to each of these two barriers. Let $y_{l}$ and $y_{u}$ be the respective lower barrier and upper barrier of the process $Y_{t}$, where $y_{l}<Y_{t_{0}}<y_{u}$; and let $\tau_{y_{l}}$ and $\tau_{y_{u}}$ denote the first passage time of $Y_{t}$ hitting $y_{l}$ and $y_{u}$, respectively. We define the following conditional joint density functions:

$$
\begin{gathered}
f_{l}(s, \xi) d s d \xi=Q\left(\tau_{y_{l}} \in(s, s+d s), \tau_{y_{l}}<\tau_{y_{u}}, X_{\tau_{y_{l}}} \in(\xi, \xi+d \xi) \mid X_{t_{0}}=x_{0}, Y_{t_{0}}=y_{0}\right) \\
f_{u}(s, \xi) d s d \xi=Q\left(\tau_{y_{u}} \in(s, s+d s), \tau_{y_{u}}<\tau_{y_{l}}, X_{\tau_{y_{u}}} \in(\xi, \xi+d \xi) \mid X_{t_{0}}=x_{0}, Y_{t_{0}}=y_{0}\right) .
\end{gathered}
$$

By the strong Markov property of the joint process of $X_{t}$ and $Y_{t}$, and considering the disjoint events $\left\{\tau_{y_{u}}<\tau_{y_{l}}\right\}$ and $\left\{\tau_{y_{l}}<\tau_{y_{u}}\right\}$, we deduce the following pair of integral equations for the conditional first passage time density functions:

$$
\begin{aligned}
& L\left(x, y, t ; x_{0}, y_{0}, t_{0}\right) \\
= & \int_{t_{0}}^{t} \int_{-\infty}^{\infty} f_{l}(s, \xi) L\left(x, y, t ; \xi, y_{l}, s\right) d \xi d s+\int_{t_{0}}^{t} \int_{-\infty}^{\infty} f_{u}(s, \xi) L\left(x, y, t ; \xi, y_{u}, s\right) d \xi d s ;
\end{aligned}
$$


and

$$
\begin{aligned}
& M\left(x, y, t ; x_{0}, y_{0}, t_{0}\right) \\
= & \int_{t_{0}}^{t} \int_{-\infty}^{\infty} f_{l}(s, \xi) M\left(x, y, t ; \xi, y_{l}, s\right) d \xi d s+\int_{t_{0}}^{t} \int_{-\infty}^{\infty} f_{u}(s, \xi) M\left(x, y, t ; \xi, y_{u}, s\right) d \xi d s .
\end{aligned}
$$

In the next section, we derive the recursive schemes for calculating the numerical approximation of the first passage time density functions by performing discretization of the above pair of integral equations. We then show how to use the approximate density function values to compute the fair value of the Coco bond through numerical integration of the integral representation formula of the value functions shown in eqs. (2.6) and (2.7).

\section{Construction of the numerical algorithm}

The numerical algorithm for pricing the Coco bond with both mechanical conversion (onetouch trigger) and regulatory conversion (Parisian trigger) involves the following sequential steps:

1. Determination of the numerical approximation of the density functions of the first passage times to the two barriers.

2. Calculation of the price functions $V_{t}\left(X_{t}, Y_{t} ; T\right)$ and $V_{t}^{G}\left(X_{t} ; T\right)$ where $t>T-d$. When the time to expiry is less than $d$, the Parisian trigger becomes immaterial.

3. Calculation of the price functions $V_{t}\left(X_{t}, Y_{t} ; T\right)$ and $V_{t}^{G}\left(X_{t} ; T\right)$ where $0 \leq t<T-d$, when both the mechanical conversion and regulatory conversion are under active mode.

As in most numerical algorithms, it is necessary to approximate the infinite domain of $X_{t}$ by a finite computational domain $\left[x_{\min }, x_{\max }\right]$, where $x_{\min }$ and $x_{\max }$ are chosen such that the probability of $X_{t}$ staying outside the finite domain $\left[x_{\min }, x_{\max }\right]$ is negligibly small. We discretize the computational domain of $\left[x_{\min }, x_{\max }\right] \times[0, T]$ by $n_{x}$ spatial grids and $n_{T}$ time steps, where $n_{x} \Delta x=x_{\max }-x_{\min }$ and $n_{T} \Delta t=T$. Here, $\Delta x$ and $\Delta T$ are the spatial stepwidth and time step, respectively. Note that the grid points in the computational domain are characterized by $\left(x_{i}, t_{j}\right)$, where $x_{i}=x_{\text {min }}+i \Delta x, i=0,1, \ldots, n_{x}$ and $t_{j}=j \Delta t, j=0,1, \ldots, n_{T}$.

First, we would like to derive the recursive schemes that compute the numerical approximation of the density/ distribution functions of the first passage times at varying values of the stock price. This is done by performing discretization of the pair of integral equations for the first passage time density functions. We define the following discrete grid values for the first passage time density/ distribution functions as numerical approximation of their continuous counterparts:

(i) $q(m, i ; y) \approx Q\left(\tau_{y} \in\left(t_{m}, t_{m}+\Delta t\right), X_{\tau_{y}} \in\left(x_{i}, x_{i}+\Delta x\right) \mid X_{0}, Y_{0}\right), \quad Y_{0}>y$;

(ii) $q^{l}(i ; y) \approx Q\left(\tau_{y}>t_{l}, X_{t_{l}} \in\left(x_{i}, x_{i}+\Delta x\right) \mid X_{0}, Y_{0}\right), \quad t_{l}>0$

(iii) $q_{y_{1}<y_{2}}(m, i) \approx Q\left(\tau_{y_{1}} \in\left(t_{m}, t_{m}+\Delta t\right), \tau_{y_{1}}<\tau_{y_{2}}, X_{\tau_{y_{1}}} \in\left(x_{i}, x_{i}+\Delta x\right) \mid X_{0}, Y_{0}\right), \quad Y_{0} \in$ $\left(y_{1}, y_{2}\right)$; 
(iv) $q_{y_{2}<y_{1}}(m, i) \approx Q\left(\tau_{y_{2}} \in\left(t_{m}, t_{m}+\Delta t\right), \tau_{y_{2}}<\tau_{y_{1}}, X_{\tau_{y_{2}}} \in\left(x_{i}, x_{i}+\Delta x\right) \mid X_{0}, Y_{0}\right), \quad Y_{0} \in$ $\left(y_{1}, y_{2}\right)$

(v) $q^{d}\left(i ; y_{1}, y_{2}\right) \approx Q\left(\min \left(\tau_{y_{1}}, \tau_{y_{2}}\right)>d, X_{d} \in\left(x_{i}, x_{i}+\Delta x\right) \mid X_{0}, Y_{0}\right), \quad Y_{0} \in\left(y_{1}, y_{2}\right)$.

Here we do not state explicitly the dependence of these numerical density and distribution functions on the initial values $X_{0}$ and $Y_{0}$ for notational simplicity.

\section{Single-barrier discrete first passage time density and distribution}

The first two discrete first passage time density and distribution function, $q(m, i ; y)$ and $q^{l}(i ; y)$, involve only single barrier $y$, so one may employ a similar approach of that of the recursive Fortet scheme proposed by Bernard et al. (2008). We take $t_{0}=0$ and assume $Y_{0}>y$, and suppose $Y_{t}$ falls below $y$ at some later time $t_{l}>0$, then $Y_{t}$ must hit the barrier $y$ at some time $\tau_{y}$ earlier than $t_{l}$. By the strong Markov property of the joint process $X_{t}$ and $Y_{t}$, and using the law of total probability, we obtain

$$
\begin{aligned}
& L\left(x_{i}, y, t_{l} ; x_{0}, y_{0}, t_{0}\right) d x \\
= & \int_{0}^{t_{l}} \int_{-\infty}^{\infty} Q\left(\tau_{y}=u, X_{u}=\xi \mid X_{0}=x_{0}, Y_{0}=y_{0}\right) \\
= & \int_{0}^{t_{l}} \int_{-\infty}^{\infty} Q\left(Y_{t_{l}}<y, X_{t_{l}} \in\left(x_{i}, x_{i}+d x\right) \mid X_{u}=\xi, Y_{u}=y\right) d \xi d u
\end{aligned}
$$

By performing discretization of the above integral equation, we have

$$
L\left(x_{i}, y, t_{l} ; x_{0}, y_{0}, t_{0}\right) \approx \sum_{m=0}^{l} \sum_{k=0}^{n_{x}} q(m, k ; y) L\left(x_{i}, y, t_{l} ; x_{k}, y, t_{m}\right) .
$$

Recall that $L\left(x_{i}, y, t_{l} ; x_{0}, y_{0}, t_{0}\right)$ admits closed form formula [see eq. (2.8a)]. It is desirable to derive a recursive scheme to determine $q(m, i ; y)$ over successive time level $m$ and at varying values of $x_{i}$.

At initiation, it is obvious that $q(0, i ; y)=0$ since $L\left(x_{i}, y, t_{0} ; x_{0}, y_{0}, t_{0}\right)=0$ for $y_{0} \neq y$. Suppose we have obtained values for $q(m, i ; y), m=0,1, \ldots, l$, we would like to derive a recursive scheme to determine $q(l+1, i ; y)$ at varying values of $i$. By virtue of the discretized scheme in eq. (3.2), we deduce that

$$
\begin{aligned}
L\left(x_{i}, y, t_{l+1} ; x_{0}, y_{0}, t_{0}\right) \approx & \sum_{m=0}^{l} \sum_{k=0}^{n_{x}} q(m, k ; y) L\left(x_{i}, y, t_{l+1} ; x_{k}, y, t_{m}\right) \\
& +\sum_{k=0}^{n_{x}} q(l+1, k ; y) L\left(x_{i}, y, t_{l+1} ; x_{k}, y, t_{l+1}\right) .
\end{aligned}
$$

Note that

$$
L\left(x_{i}, y, t_{l+1} ; x_{k}, y, t_{l+1}\right)=\left\{\begin{array}{ll}
1 & \text { if } k=i \\
0 & \text { otherwise }
\end{array} ;\right.
$$


so we obtain the following recursive formula for $q(l+1, i ; y)$ :

$$
q(l+1, i ; y)=L\left(x_{i}, y, t_{l+1} ; x_{0}, y_{0}, t_{0}\right)-\sum_{m=0}^{l} \sum_{k=0}^{n_{x}} q(m, k ; y) L\left(x_{i}, y, t_{l+1} ; x_{k}, y, t_{m}\right) .
$$

Next, we show how to determine $q^{l}(i ; y)$ in terms of $q(m, i ; y)$ and $M\left(x_{i}, y, t_{l} ; x_{0}, y_{0}, t_{0}\right)$. Suppose $Y_{0}>y$ while $Y_{t_{l}}>y$, then either $Y_{t}$ hits the barrier $y$ from above before time $t_{l}$ or $Y_{t}$ never hits the barrier between times 0 and $t_{l}$. Again, by the strong Markov property of the joint process of $X_{t}$ and $Y_{t}$, conditioning on the first passage time $\tau_{y}$, we obtain

$$
\begin{aligned}
& M\left(x_{i}, y, t_{l} ; x_{0}, y_{0}, t_{0}\right) d x \\
= & \int_{0}^{t_{l}} \int_{-\infty}^{\infty} Q\left(\tau_{y}=u, X_{u}=\xi \mid X_{0}=x_{0}, Y_{0}=y_{0}\right) \\
& Q\left(Y_{t_{l}}>y, X_{t_{l}} \in\left(x_{i}, x_{i}+d x\right) \mid X_{u}=\xi, Y_{u}=y\right) d \xi d u \\
& +Q\left(\tau_{y}>t_{l}, X_{t_{l}} \in\left(x_{i}, x_{i}+d x\right) \mid X_{0}=x_{0}, Y_{0}=y_{0}\right) .
\end{aligned}
$$

Note that $\tau_{y}>t_{l}$ would imply $Y_{t_{l}}>y$ implicitly. By performing discretization of eq. (3.4), we obtain

$$
q^{l}(i ; y)=M\left(x_{i}, y, t_{l} ; x_{0}, y_{0}, t_{0}\right)-\sum_{m=0}^{l} \sum_{k=0}^{n_{x}} q(m, k ; y) M\left(x_{i}, y, t_{l} ; x_{k}, y, t_{m}\right)
$$

\section{Two-barrier discrete first passage time density and distribution}

To deal with the presence of two barriers in our CoCo pricing model, it is necessary to determine $q_{y_{1}<y_{2}}(m, i), q_{y_{2}<y_{1}}(m, i)$ and $q^{d}\left(i ; y_{1}, y_{2}\right)$. In a similar manner, we perform the discretization of eqs. $(2.9 \mathrm{a}, \mathrm{b})$ as follows:

$$
\begin{aligned}
L\left(x_{i}, y_{1}, t_{l} ; x_{0}, y_{0}, t_{0}\right)= & \sum_{m=0}^{l} \sum_{k=0}^{n_{x}} q_{y_{1}<y_{2}}(m, k) L\left(x_{i}, y_{1}, t_{l} ; x_{k}, y_{1}, t_{m}\right) \\
& +\sum_{m=0}^{l} \sum_{k=0}^{n_{x}} q_{y_{2}<y_{1}}(m, k) L\left(x_{i}, y_{1}, t_{l} ; x_{k}, y_{2}, t_{m}\right) ; \\
M\left(x_{i}, y_{2}, t_{l} ; x_{0}, y_{0}, t_{0}\right)= & \sum_{m=0}^{l} \sum_{k=0}^{n_{x}} q_{y_{1}<y_{2}}(m, k) M\left(x_{i}, y_{2}, t_{l} ; x_{k}, y_{1}, t_{m}\right) \\
& +\sum_{m=0}^{l} \sum_{k=0}^{n_{x}} q_{y_{2}<y_{1}}(m, k) M\left(x_{i}, y_{2}, t_{l} ; x_{k}, y_{2}, t_{m}\right) ;
\end{aligned}
$$

where $y_{0} \in\left(y_{1}, y_{2}\right)$.

Again, we would like to construct the recursive schemes to determine $q_{y_{1}<y_{2}}(l, i)$ at successive time levels $t_{l}, l=0,1, \ldots$ and varying values of $x_{i}, i=0,1, \ldots, n_{x}$. At initiation, we deduce that

$$
\begin{aligned}
& q_{y_{1}<y_{2}}(0, i)=L\left(x_{i}, y_{1}, t_{0} ; x_{0}, y_{0}, t_{0}\right)=0 \\
& q_{y_{2}<y_{1}}(0, i)=M\left(x_{i}, y_{2}, t_{0} ; x_{0}, y_{0}, t_{0}\right)=0
\end{aligned}
$$


Given the values of $q_{y_{1}<y_{2}}(k, i)$ and $q_{y_{2}<y_{1}}(k, i)$ for $k=0,1, \ldots, m$, we would like to derive the recursive scheme to determine $q_{y_{1}<y_{2}}(m+1, i)$ and $q_{y_{2}<y_{1}}(m+1, i)$. By observing the following relations:

$$
M\left(x_{m}, y_{2}, t_{l+1} ; x_{k}, y_{1}, t_{l+1}\right)=0
$$

and

$$
M\left(x_{m}, y_{2}, t_{l+1} ; x_{k}, y_{2}, t_{l+1}\right)= \begin{cases}1 & \text { if } k=m \\ 0 & \text { otherwise }\end{cases}
$$

and following a similar technique used in the single-barrier case, we obtain

$$
\begin{aligned}
q_{y_{1}<y_{2}}(m+1, i)= & L\left(x_{i}, y_{1}, t_{m+1} ; x_{0}, y_{0}, t_{0}\right)-\sum_{k=0}^{m} \sum_{j=0}^{n_{x}} q_{y_{2}<y_{1}}(k, j) L\left(x_{i}, y_{1}, t_{m+1} ; x_{j}, y_{2}, t_{k}\right) \\
& -\sum_{k=0}^{m} \sum_{j=0}^{n_{x}} q_{y_{1}<y_{2}}(k, j) L\left(x_{i}, y_{1}, t_{m+1} ; x_{j}, y_{1}, t_{k}\right)
\end{aligned}
$$

and

$$
\begin{aligned}
q_{y_{2}<y_{1}}(m+1, i)= & M\left(x_{i}, y_{2}, t_{m+1} ; x_{0}, y_{0}, t_{0}\right)-\sum_{k=0}^{m} \sum_{j=0}^{n_{x}} q_{y_{2}<y_{1}}(k, j) M\left(x_{i}, y_{2}, t_{m+1} ; x_{j}, y_{2}, t_{k}\right) \\
& -\sum_{k=0}^{m} \sum_{j=0}^{n_{x}} q_{y_{1}<y_{2}}(k, j) M\left(x_{i}, y_{2}, t_{m+1} ; x_{j}, y_{1}, t_{k}\right)
\end{aligned}
$$

Lastly, in order to derive a numerical approximation scheme for finding $q^{d}\left(i ; y_{1}, y_{2}\right)$ where $Y_{0} \in\left(y_{1}, y_{2}\right)$, we observe that the logarithm of the capital ratio $Y_{d}$ at a later time $d$ stays within $\left(y_{1}, y_{2}\right)$ provided that (i) $Y_{t} \in\left(y_{1}, y_{2}\right)$ for $0 \leq t \leq d$, (ii) $\tau_{y_{1}}<\min \left\{\tau_{y_{2}}, d\right\}$ and $Y_{d} \in\left(y_{1}, y_{2}\right)$, (iii) $\tau_{y_{2}}<\min \left\{\tau_{y_{1}}, d\right\}$ and $Y_{d} \in\left(y_{1}, y_{2}\right)$. Again, by the strong Markov property of the joint process $X_{t}$ and $Y_{t}$ and using the law of total probability, we obtain

$$
\begin{aligned}
& Q\left(Y_{d} \in\left(y_{1}, y_{2}\right), X_{d} \in\left(x_{i}, x_{i}+d x\right) \mid X_{0}, Y_{0}\right) \\
= & Q\left(\min \left\{\tau_{y_{1}}, \tau_{y_{2}}\right\}>d, X_{d} \in\left(x_{i}, x_{i}+d x\right) \mid X_{0}, Y_{0}\right) \\
+ & \int_{0}^{d} \int_{-\infty}^{\infty} Q\left(\tau_{y_{1}}=u, \tau_{y_{1}}<\tau_{y_{2}}, X_{u}=\xi \mid X_{0}, Y_{0}\right) \\
& +\int_{0}^{d} \int_{-\infty}^{\infty} Q\left(Y_{d} \in\left(y_{1}, y_{2}\right), X_{d} \in\left(x_{i}, x_{i}+d x\right)\left|X_{u}=\xi, Y_{u}=y_{y_{2}}<\tau_{y_{1}}, X_{u}=\xi\right| X_{0}, Y_{0}\right) \\
& Q\left(Y_{d} \in\left(y_{1}, y_{2}\right), X_{d} \in\left(x_{i}, x_{i}+d x\right) \mid X_{u}=\xi, Y_{u}=y_{2}\right) d \xi d u .
\end{aligned}
$$


Let $n_{d}$ be the number of time steps corresponding to the time period $d$, where $n_{d} \Delta t=d$. By discretization of the above equation, we obtain

$$
\begin{aligned}
q^{d}\left(i ; y_{1}, y_{2}\right)= & L\left(x_{i}, y_{2}, d ; X_{0}, Y_{0}, t_{0}\right)-L\left(x_{i}, y_{1}, d ; X_{0}, Y_{0}, t_{0}\right) \\
& -\sum_{m=0}^{n_{d}} \sum_{j=0}^{n_{x}} q_{y_{1}<y_{2}}(m, j)\left[L\left(x_{i}, y_{2}, d ; x_{j}, y_{1}, t_{m}\right)-L\left(x_{i}, y_{1}, d ; x_{j}, y_{1}, t_{m}\right)\right] \\
& -\sum_{m=0}^{n_{d}} \sum_{j=0}^{n_{x}} q_{y_{2}<y_{1}}(m, j)\left[L\left(x_{i}, y_{2}, d ; x_{j}, y_{2}, t_{m}\right)-L\left(x_{i}, y_{1}, d ; x_{j}, y_{2}, t_{m}\right)\right] .
\end{aligned}
$$

\section{Numerical approximation of the price functions}

The price function $V_{t}\left(X_{t}, Y_{t} ; T\right)$ can be represented in an integral form as shown in eq. (2.6), which involves the auxiliary value function $V_{u}^{G}(x ; T)$, conditional joint density $f_{G}^{-}(u, x)$ and marginal density function $F_{G}^{-}(x)$. For $t+d<T$, the auxiliary value function $V_{t}^{G}\left(X_{t} ; T\right)$ admits the integral representation [see eq. (2.7)] that involves the marginal density function $F_{G^{+}, B}(x)$ and conditional density functions $f_{G^{+}}(u, x)$ and $f_{B}(u, x)$.

Recall from eq. (2.4) that $V_{t}^{G}\left(X_{t} ; T\right)$ and $V_{t}\left(X_{t}, Y_{t} ; T\right)$ are related to each other by taking $Y_{t} \rightarrow \ln G-\varepsilon$, where $\varepsilon \rightarrow 0^{+}$, under the continuous time formulation. We assume that the regulatory authority would initiate monitoring of the issuing bank when the issuer's capital ratio falls slightly below $G$ and the monitoring phase is terminated when the capital ratio rises slightly above $G$. In our numerical algorithm, we choose a finite small positive constant $\varepsilon$ such that the monitoring phase is initiated when $Y_{t}$ moves down to $\ln G-\varepsilon$ and monitoring is terminated when $Y_{t}$ rises above $\ln G+\varepsilon$. We are then interested to find numerical approximation to the price functions $V_{t}\left(X_{t}, \ln G+\varepsilon ; T\right)$ and $V_{t}^{G}\left(X_{t} ; T\right)$ in terms of the numerical approximation to the marginal density functions derived in the above. This is done by performing numerical integration of the integral formulas of $V_{t}\left(X_{t}, Y_{t} ; T\right)$ and $V_{t}^{G}\left(X_{t} ; T\right)$.

Since the Parisian trigger becomes ineffective when the time to maturity is less than $d$, or $t \geq T-d$, it is necessary to separate our numerical calculations in the two separate cases (i) $t \geq T-d$ and (ii) $0 \leq t<T-d$. In our subsequent exposition, we write $\left.q(m, i ; y)\right|_{X_{0}, Y_{0}}$, $\left.q^{l}(i, y)\right|_{X_{0}, Y_{0}}$, etc. to specify the initial values for $X_{t}$ and $Y_{t}$ to be $X_{0}$ and $Y_{0}$, respectively for better clarity.

(i) $t \geq T-d$

Suppose the discrete time level $t_{m} \in[T-d, T]$, we then have

$$
\begin{aligned}
V_{t_{m}}\left(x_{i}, \ln G+\varepsilon ; T\right) \approx & \left.\sum_{l=m}^{n_{T}} \sum_{j=0}^{n_{x}} q(l-m, j ; \ln B)\right|_{x_{i}, \ln G+\varepsilon}\left[c\left(t_{m}, t_{l}\right)+e^{-r\left(t_{l}-t_{m}\right)} N \max \left\{e^{x_{j}}, K\right\}\right] \\
& +\left.\sum_{j=0}^{n_{x}} q^{n_{T}-m}(j ; \ln B)\right|_{x_{i}, \ln G+\varepsilon}\left[c\left(t_{m}, T\right)+e^{-r\left(T-t_{m}\right)} F\right]
\end{aligned}
$$


and

$$
\begin{aligned}
V_{t_{m}}^{G}\left(x_{i} ; T\right) \approx & \left.\sum_{l=m}^{n_{T}} \sum_{j=0}^{n_{x}} q(l-m, j ; \ln B)\right|_{x_{i}, \ln G-\varepsilon}\left[c\left(t_{m}, t_{l}\right)+e^{-r\left(t_{l}-t_{m}\right)} N \max \left\{e^{x_{j}}, K\right\}\right] \\
& +\left.\sum_{j=0}^{n_{x}} q^{n_{T}-m}(j ; \ln B)\right|_{x_{i}, \ln G-\varepsilon}\left[c\left(t_{m}, T\right)+e^{-r\left(T-t_{m}\right)} F\right] .
\end{aligned}
$$

(ii) $0 \leq t<T-d$

Suppose the time level $t_{m} \in[0, T-d]$, we have

$$
\begin{aligned}
& V_{t_{m}}\left(x_{i}, \ln G+\varepsilon ; T\right) \\
\approx & \left.\sum_{l=m}^{n_{T}} \sum_{j=0}^{n_{x}} q(l-m, j ; \ln G-\varepsilon)\right|_{x_{i}, \ln G+\varepsilon}\left[c\left(t_{m}, t_{l}\right)+e^{-r\left(t_{l}-t_{m}\right)} V_{t_{l}}^{G}\left(x_{j} ; T\right)\right] \\
& +\left.\sum_{j=0}^{n_{x}} q^{n_{T}-m}(j ; \ln G-\varepsilon)\right|_{x_{i}, \ln G+\varepsilon}\left[c\left(t_{m}, T\right)+e^{-r\left(T-t_{m}\right)} F\right]
\end{aligned}
$$

and

$$
\begin{aligned}
& \quad V_{t_{m}}^{G}\left(x_{i} ; T\right) \\
& \left.\approx \sum_{l=m}^{m+n_{d}} \sum_{j=0}^{n_{x}} q_{\ln B<\ln G+\varepsilon}(l-m, j)\right|_{x_{i}, \ln G-\varepsilon}\left[c\left(t_{m}, t_{l}\right)+e^{-r\left(t_{l}-t_{m}\right)} N \max \left\{e^{x_{j}}, K\right\}\right] \\
& \quad+\left.\sum_{l=m}^{m+n_{d}} \sum_{j=0}^{n_{x}} q_{\ln G+\varepsilon<\ln B}(l-m, j)\right|_{x_{i}, \ln G-\varepsilon}\left[c\left(t_{m}, t_{l}\right)+e^{-r\left(t_{l}-t_{m}\right)} V_{t_{l}}\left(x_{j}, \ln G+\varepsilon ; T\right)\right] \\
& \quad+\left.\sum_{j=0}^{n_{x}} q^{d}(j ; \ln B, \ln G+\varepsilon)\right|_{x_{i}, \ln G-\varepsilon}\left[c\left(t_{m}, t_{m}+d\right)+e^{-r d} N \max \left\{e^{x_{j}}, K\right\}\right] .
\end{aligned}
$$

In particular, the numerical approximation to the price function at initiation $V_{0}\left(X_{0}, Y_{0} ; T\right)$ is given by

$$
\begin{aligned}
V_{0}\left(X_{0}, Y_{0} ; T\right) \approx & \left.\sum_{l=0}^{n_{T}} \sum_{j=0}^{n_{x}} q(l, j ; \ln G-\varepsilon)\right|_{X_{0}, Y_{0}}\left[c\left(t_{0}, t_{l}\right)+e^{-r t_{l}} V_{t_{l}}^{G}\left(x_{j} ; T\right)\right] \\
& +\left.\sum_{j=0}^{n_{x}} q^{n_{T}}(j ; \ln G-\varepsilon)\right|_{X_{0}, Y_{0}}\left[c\left(t_{0}, T\right)+e^{-r\left(T-t_{0}\right)} F\right] .
\end{aligned}
$$

\section{Numerical results}

In this section, we present numerical test results on the examination of accuracy of numerical results for the price function of the Coco bond and analyze the order of convergence of the Fortet algorithm. We also compare the Fortet algorithm with the Monte Carlo simulation method with regard to accuracy and run time efficiency. Lastly, we examine the pricing behavior of the Coco bonds under various market conditions. 


\begin{tabular}{|l|l|l|l|}
\hline $\begin{array}{l}\text { Number of time } \\
\text { steps, } n_{T}\end{array}$ & $\begin{array}{l}\text { Number of } x- \\
\text { grids, } n_{x}\end{array}$ & $\begin{array}{l}\text { Numerical value } \\
\text { of price function }\end{array}$ & $\begin{array}{l}\text { CPU time in } \\
\text { seconds) }\end{array}$ \\
\hline \multirow{3}{*}{252} & 50 & 1050.3103 & 20.511 \\
& 100 & 1050.0603 & 102.486 \\
\hline \multirow{3}{*}{504} & 200 & 1050.0550 & 638.760 \\
& 50 & 1035.3322 & 64.916 \\
& 100 & 1035.1232 & 345.698 \\
& 200 & 1035.1224 & 2851.536 \\
\hline
\end{tabular}

Table 1: Impact of number of $x$-grids $n_{x}$ and time steps $n_{T}$ in the Fortet algorithm on the numerical value of the price function and the CPU time. The CPU time required increases roughly by a factor of $5-8$ when $n_{x}$ is doubled and a factor of 3 when $n_{T}$ is doubled.

\subsection{Numerical accuracy, order of convergence and computational efficiency of the Fortet algorithm}

First, we would like to examine the discretization errors arising from spatial and temporal discretization of the computational domain. The parameter values used in our numerical calculations were chosen to be: $r=0.03, q=0.025, \sigma=0.25, \sigma_{Y}=0.5, \rho=0.3, \alpha=1.0488$, $\mu=\ln 0.1, T=2, F=1000, c=0.08, K=2.5, d=\frac{15}{63}, N=100, G=0.075, B=0.05$. In Table 1, we present the numerical values of the price function of the Coco bond computed using varying number of grids $n_{x}$ in the spatial $x$-domain and number of time steps $n_{T}$ in the temporal domain. We observe that the change in numerical value of the price function is almost insignificant with respect to different choices of $n_{x}$. However, the choices of $n_{T}$ have more significant impact on numerical accuracy of the price function. This is because a fine resolution of the first passage time is required for good numerical accuracy. In terms of computational costs, the CPU time required increases roughly by a factor of 5 to 8 when $n_{x}$ is doubled and a factor of 3 when $n_{T}$ is doubled.

Next, we performed another set of numerical tests to examine the order of convergence of the Fortet algorithm with respect to the number of time steps $n_{T}$. Almost the same set of parameter values in Table 1 are used to generate the numerical values of the price function in Table 2, except that $n_{x}$ is fixed to be 50 . The numerical value of the price function of 1016.5654 was obtained by performing 10 million simulation paths in Monte Carlo simulation (used as benchmark comparison for numerical accuracy). In Table 2, we observe that the ratio of the relative error is very close to 2. This indicates that the Fortet algorithm achieves first order temporal accuracy.

\subsection{Pricing behavior of the Coco bonds}

We would like to examine the pricing behavior of the Coco bonds under various market conditions. We examine the dependence of the price function of the Coco bond, $V_{0}\left(X_{0}, Y_{0} ; T\right)$, on $X_{0}, Y_{0}$ and $T$. In Figure 1 , we show the plot of $V_{0}\left(X_{0}, Y_{0} ; T\right)$ against $X_{0}$. The parameter values used in the numerical calculations for the base case are: $r=0.03, q=0.025, \mu=\ln 0.1$, 


\begin{tabular}{|l|l|l|l|}
\hline $\begin{array}{l}\text { Number of time } \\
\text { steps, } n_{T}\end{array}$ & $\begin{array}{l}\text { Numerical value } \\
\text { of price function }\end{array}$ & $\begin{array}{l}\text { Relative per- } \\
\text { centage error }\end{array}$ & $\begin{array}{l}\text { Ratio of differ- } \\
\text { ence in values }\end{array}$ \\
\hline 252 & 1050.3103 & $3.3195 \%$ & \\
\hline 504 & 1035.3322 & $1.8461 \%$ & \\
\hline 1008 & 1025.9632 & $0.9245 \%$ & 1.5987 \\
\hline 2016 & 1020.8578 & $0.4222 \%$ & 1.8351 \\
\hline 4032 & 1018.2806 & $0.1687 \%$ & 1.9810 \\
\hline 8064 & 1017.0192 & $0.0446 \%$ & 2.0431 \\
\hline
\end{tabular}

Table 2: The difference between two consecutive iterations increases roughly by a factor of two when the number of time steps is halved, indicating first order temporal accuracy of the Fortet algorithm. The benchmark Monte Carlo simulation result using 10 million simulation paths is 1016.5654 .

$\sigma=0.25, \sigma_{Y}=0.5, \rho=0.3, \alpha=1.0488, d=\frac{15}{63}, T=2, F=1000, c=0.08, N=100$, $K=2.5, G=0.075, B=0.05$ and $Y_{0}=\ln 0.12$. As expected, the price function increases monotonically with respect to $X_{0}$. Since the Coco bond has a relatively high bond component and low level of optionality in equity payoff, the impact of interest rate $r$ on the price function is more significant compared to the stock price volatility $\sigma$ and correlation coefficient $\rho$ of the underlying joint process of $X_{t}$ and $Y_{t}$. Since the trigger conditions for conversion are highly dependent on $Y_{t}$, the capital ratio volatility $\sigma_{Y}$ has a stronger influence on the price function compared to the stock price volatility $\sigma$. Next, we show the plot of $V_{0}\left(X_{0}, Y_{0} ; T\right)$ against $Y_{0}$ in Figure 2. The parameter values used in the numerical calculations for the base case are the same as those for Figure 1, except that $X_{0}$ is taken to be 2 . The price function is monotonically increasing with respect to $Y_{0}$, an expected result. It is seen that the mean reversion level $\mu$ has a stronger influence on the price function when compared with the mean reversion speed $\alpha$. A lower mean reversion level $\mu$ and a high mean reversion speed $\alpha$ would decrease the value of the Coco bond since the chance of equity conversion becomes higher.

It is instructive to examine the impact of $X_{0}, Y_{0}$ and $d$ on the respective probability of the Parisian trigger and mechanical trigger (see Table 3). The numerical results were obtained using Monte Carlo calculations with 16,128 time steps and 1 million simulation paths. The parameter values used in the calculations for the base case are the same as those used for generating the plots in Figure 1. As revealed from the results in Table 3, the level of $Y_{0}$ and excursion time factor $d$ have relatively strong influence on the probability of the Parisian trigger and mechanical trigger. When $d$ is chosen to be relatively small, signifying stronger regulatory monitoring on the financial state of the issuing bank, the probability of the Parisian trigger increases quite significantly while that of the mechanical trigger becomes smaller. As confirmed from the numerical results in Table 3, the stock price level $X_{0}$ has almost insignificant impact on the probabilities of equity conversion. This is not surprising since equity conversion is expected to be mostly dependent on the capital ratio. 


\begin{tabular}{|l|l|l|l|l|}
\hline$X_{0}$ & $Y_{0}$ & $d$ & $\begin{array}{l}\text { Probability of } \\
\text { Parisian trigger }\end{array}$ & $\begin{array}{l}\text { Probability of } \\
\text { mechanical trigger }\end{array}$ \\
\hline 2 & $\ln 0.15$ & $d=\frac{15}{63}$ & 0.0979 & 0.0567 \\
\hline 2 & $\ln 0.12$ & $d=\frac{15}{63}$ & 0.1339 & 0.0762 \\
\hline 2 & $\ln 0.09$ & $d=\frac{5}{63}$ & 0.2126 & 0.1183 \\
\hline 1.5 & $\ln 0.12$ & $d=\frac{15}{63}$ & 0.1345 & 0.0765 \\
\hline 2 & $\ln 0.12$ & $d=\frac{15}{63}$ & 0.1339 & 0.0762 \\
\hline 2.5 & $\ln 0.12$ & $d=\frac{15}{63}$ & 0.1340 & 0.0762 \\
\hline 2 & $\ln 0.12$ & $d=\frac{10}{63}$ & 0.2301 & 0.0427 \\
\hline 2 & $\ln 0.12$ & $d=\frac{5}{63}$ & 0.3678 & 0.0058 \\
\hline
\end{tabular}

Table 3: Probability of the Parisian trigger and mechanical trigger at varying level of $X_{0}, Y_{0}$ and $d$.

\section{Conclusion}

We propose a two-state pricing model of the CoCo bond using the joint process of the stock price and Tier 1 capital ratio. The accounting trigger of equity conversion into shares of the bond issuer is activated when the capital ratio hits a lower threshold value while the regulatory trigger is activated when the capital ratio stays under the non-viable state cumulatively for a certain period of time. The dimensionality of the CoCo bond pricing model increases by one due to the inclusion of an extra Parisian state variable that models the regulatory trigger mechanism. In this paper, we construct an effective numerical algorithm that avoids the nuisance of introducing an additional path dependent state variable for the Parisian feature of regulatory trigger. The design of the algorithm is based on the extended Fortet method that solves a pair of integral equations for the determination of the random first passage times of the capital ratio process to the upper Parisian threshold (warning threshold of non-viability) and the lower one-touch knock-out threshold. Dimension reduction in the pricing algorithm is achieved via the determination of the auxiliary CoCo bond function at the Tier 1 capital ratio that is slightly below the warning threshold of non-viability. As revealed from our numerical experiments, our numerical algorithm based on the Fortet method is seen to compete favorably well in terms of accuracy and runtime efficiency when compared with the Monte Carlo simulation method. Pricing properties of the CoCo bond price function under various model parameter values are presented. The volatility parameters of the stock price process and capital ratio process have relatively small influence on the price function when compared with the level of interest rate, indicating the dominant bond nature of the Coco bond. The probability of the Parisian trigger and mechanical trigger are strongly dependent on the level of capital ratio and the length of the excursion time period staying within the non-viable state. 


\section{Reference}

Avdjiev, S., Kartasheva, A., Bogdanova, B., 2013. CoCos: a primer. BIS Quarterly Review, 43-56.

Bernard, C., Le Courtois, O., Quittard-Pinon, F., 2005. Market value of life insurance contracts under stochastic interest rates and default risk. Insurance: Mathematics and Economics, 36, 499-516.

Bernard, C., Le Courtois, O., Quittard-Pinon, F., 2006. Development and pricing of a new participating contract. North American Actuarial Journal, 10(4), 179-195.

Bernard, C., Le Courtois, O., Quittard-Pinon, F., 2008. Pricing derivatives with barriers in a stochastic interest rate environment. Journal of Economic and Dynamic Control, 32, 2903-2938.

Brigo, D., Garcia, J., Pede, N., 2015. CoCo bond pricing with credit and equity calibrated first-passage firm value models. International Journal of Theoretical and Applied Finance, 18(3), 1550015 (31 pages).

Cheriditio, P., Xu, Z., 2014. A reduced form CoCo model with deterministic conversion intensity. Working paper of Princeton University.

Coculescu, D., Geman, H., Jeanblanc, M., 2008. Valuation of default-sensitive claims under imperfect information. Finance and Stochastics 12, 195-218.

Collin-Dufresne, P., Goldstein, R.S., 2001. Do credit spreads reflect stationary leverage ratios? Journal of Finance, 56 (5), 1929-1957.

De Spiegeleer, J., Schoutens, W., 2012. Pricing contingent convertibles: A derivatives approach. Journal of Derivatives, 27-36.

Glasserman, P., Nouri, B., 2012. Contingent capital with a capital-ratio trigger. Management Science, 58(10), 1816-1833.

Kwok, Y.K., 2011. Lattice tree methods for strongly path dependent options. Encyclopedia of Quantitative Finance, edited by Cont. R., John Wiley and Sons Ltd, Chichester, United Kingdom, 1022-1027.

Longstaff, F., Schwartz, E., 1995. A simple approach to valuing risky fixed and floating rate debt. Journal of Finance, 50(3), 789-820.

Peloger, M., 2012. Contingent convertible bonds: pricing, dilution costs and efficient regulation. Working paper of University of California at Berkeley.

Wilkens, S., Bethke, N., 2014. Contingent convertible (CoCo) bonds: A first empirical assessment of selected pricing models. Financial Analysts Journal, 70(2), 59-77. 


\section{Appendix A - Derivation of eq. (2.8a)}

Starting from the dynamic equation for $X_{t}$ :

$$
d X_{t}=\left(r-q-\frac{\sigma^{2}}{2}\right) d t+\sigma\left(\sqrt{1-\rho^{2}} d W_{t}^{1}+\rho d W_{t}^{2}\right)
$$

we obtain

$$
\begin{aligned}
& \mathbb{E}\left[X_{t} \mid X_{t_{0}}=x_{0}\right]=x_{0}+\left(r-q-\frac{\sigma^{2}}{2}\right)\left(t-t_{0}\right), \\
& \operatorname{var}\left(X_{t} \mid X_{t_{0}}=x_{0}\right)=\sigma^{2}\left(t-t_{0}\right) .
\end{aligned}
$$

Similarly, the mean and variance of the following mean reversion process

$$
d Y_{t}=\alpha\left(\mu-Y_{t}\right) d t+\sigma_{Y} d W_{t}^{2}
$$

are found to be

$$
\begin{aligned}
& \mathbb{E}\left[Y_{t} \mid Y_{t_{0}}=y_{0}\right]=y_{0} e^{-\alpha\left(t-t_{0}\right)}+\mu\left[1-e^{-\alpha\left(t-t_{0}\right)}\right], \\
& \operatorname{var}\left(Y_{t} \mid Y_{t_{0}}=y_{0}\right)=\frac{\sigma_{Y}^{2}}{2 \alpha}\left[1-e^{-2 \alpha\left(t-t_{0}\right)}\right] .
\end{aligned}
$$

As the joint process $X_{t}$ and $Y_{t}$ is joint Gaussian, by the Projection Theorem, the mean and variance of $Y_{t} \mid X_{t}$ are given by

$$
\begin{aligned}
& \mu_{Y_{t} \mid X_{t}}=\mathbb{E}\left[Y_{t}\right]+\frac{\operatorname{cov}\left(X_{t}, Y_{t}\right)}{\operatorname{var}\left(X_{t}\right)}\left(X_{t}-\mathbb{E}\left[X_{t}\right]\right), \\
& \Sigma_{Y}^{2}=\operatorname{var}\left(Y_{t}\right)-\frac{\operatorname{cov}\left(X_{t}, Y_{t}\right)^{2}}{\operatorname{var}\left(X_{t}\right)}
\end{aligned}
$$

It is straightforward to obtain

$$
\operatorname{cov}\left(X_{t}, Y_{t} \mid X_{t_{0}}=x_{0}, Y_{t_{0}}=y_{0}\right)=\frac{\rho \sigma \sigma_{Y}}{\alpha}\left[1-e^{-\alpha\left(t-t_{0}\right)}\right]
$$

Finally, the distribution function of $Y_{t}$ conditional on varying level of $X_{t}$ is given by the product of the density function $f_{X_{t}}$ and the conditional distribution function $F_{Y_{t} \mid X_{t}}$ as follows:

$$
\begin{aligned}
L\left(x, y, t ; x_{0}, y_{0}, t_{0}\right) & =f_{X_{t}}\left(x ; x_{0}\right) F_{Y_{t} \mid X_{t}}\left(y ; y_{0}\right) \\
& =\frac{1}{\sqrt{2 \pi \operatorname{var}\left(X_{t} \mid X_{t_{0}}\right)}} e^{-\frac{\left\{x-\mathbb{E}\left[X_{t} \mid X_{t_{0}}=x_{0}\right]\right\}^{2}}{2 \operatorname{var}\left(X_{t} \mid X_{t_{0}}\right)}} \int_{-\infty}^{y} \frac{1}{\sqrt{2 \pi \Sigma_{Y}^{2}}} e^{-\frac{\left(\xi-\mu_{Y}\right)^{2}}{2 \Sigma_{Y}^{2}}} d \xi \\
& =\frac{e^{-\frac{\left[x-x_{0}-\left(r-q-\frac{\sigma^{2}}{2}\right)\left(t-t_{0}\right)\right]^{2}}{2 \sigma^{2}\left(t-t_{0}\right)}}}{\sqrt{2 \pi \sigma^{2}\left(t-t_{0}\right)}} N\left(\frac{y-\mu_{Y}}{\Sigma_{Y}}\right) .
\end{aligned}
$$




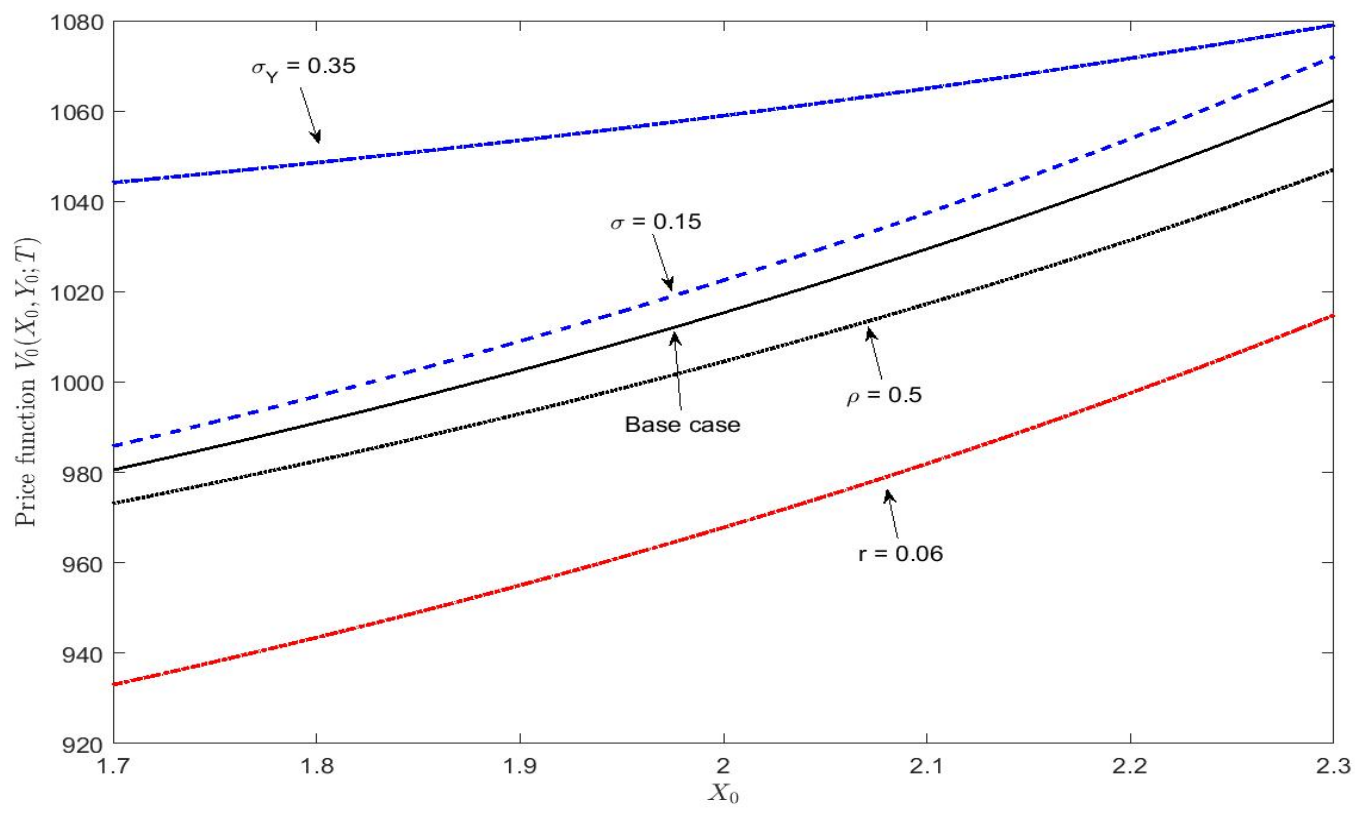

Figure 1: Plot of the price function $V_{0}\left(X_{0}, Y_{0} ; T\right)$ against $X_{0}$. The interest rate $r$ and capital ratio volatility $\sigma_{Y}$ have more significant impact on the price function when compared with the stock price volatility $\sigma$ and correlation coefficient $\rho$ of the joint process $X_{t}$ and $Y_{t}$.

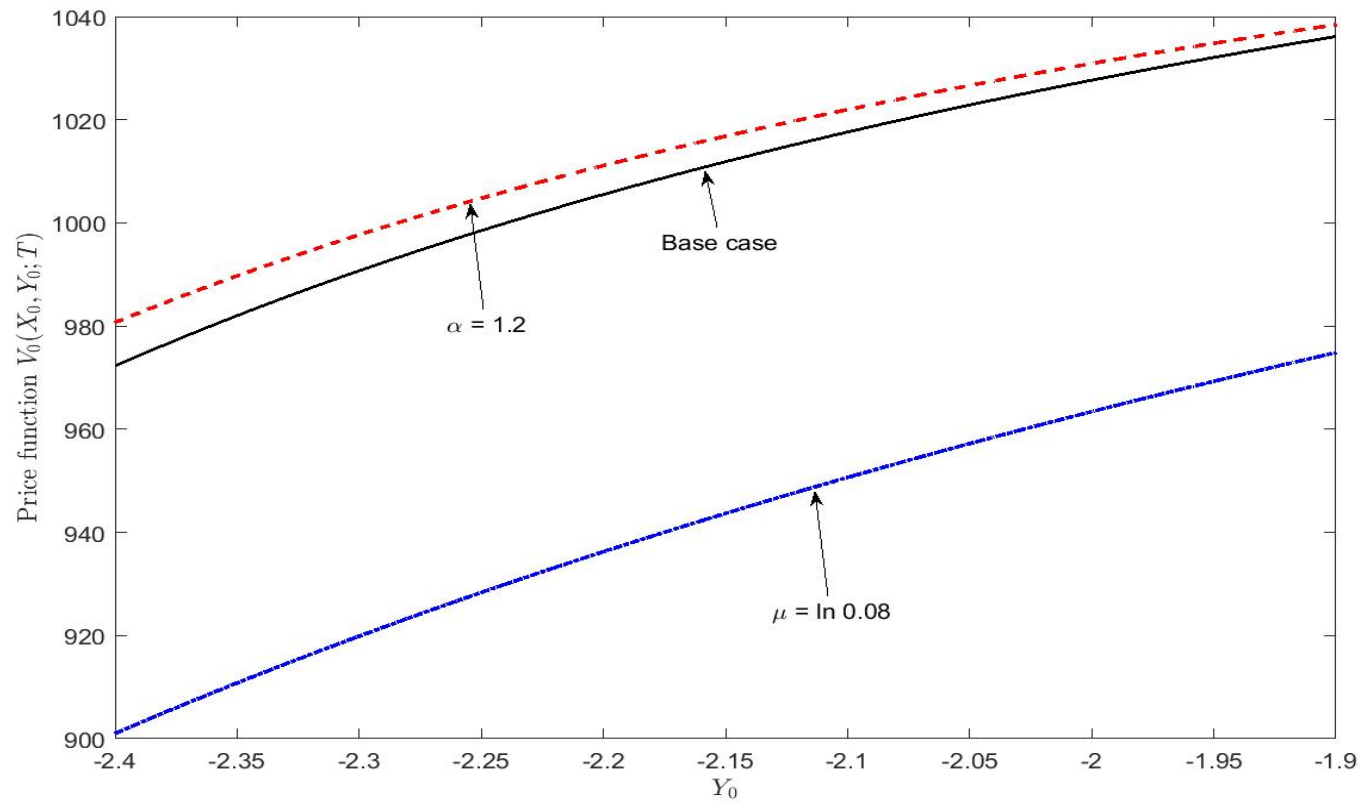

Figure 2: Plot of the price function $V_{0}\left(X_{0}, Y_{0} ; T\right)$ against $Y_{0}$. The mean reversion level of capital ratio $\mu$ shows stronger influence on the price function when compared with mean reversion speed $\alpha$. 